\title{
Transient photoluminescence enhancement as a probe of the structure of impurity-trapped excitons in $\mathrm{CaF}_{2}: \mathrm{Yb}^{2+}$
}

\author{
Michael F. Reid, ${ }^{1}$ Pubudu S. Senanayake, ${ }^{2}$ Jon-Paul R. Wells, ${ }^{2, *}$ Giel Berden,${ }^{3}$ Andries Meijerink, ${ }^{4}$ Alexander J. Salkeld ${ }^{2}$ \\ Chang-Kui Duan, ${ }^{5}$ and Roger J. Reeves ${ }^{1}$ \\ ${ }^{1}$ Department of Physics and Astronomy and MacDiarmid Institute for Advanced Materials and Nanotechnology, University of Canterbury, \\ PB 4800, Christchurch 8140, New Zealand \\ ${ }^{2}$ Department of Physics and Astronomy, University of Canterbury, PB 4800, Christchurch 8140, New Zealand \\ ${ }^{3}$ FELIX Free Electron Laser Facility, FOM Institute for Plasmaphysics Rijnhuizen, PO Box 1207, 3430 BE, Nieuwegein, The Netherlands \\ ${ }^{4}$ Debye Institute for NanoMaterials Science, University of Utrecht, P.O. Box 80000, TA 3508 Utrecht, The Netherlands \\ ${ }^{5}$ Department of Physics, University of Science and Technology of China, Hefei 230026, China \\ (Received 15 July 2011; revised manuscript received 23 August 2011; published 23 September 2011)
}

\begin{abstract}
We demonstrate a direct measurement of the energy levels of impurity-trapped excitons in $\mathrm{CaF}_{2}: \mathrm{Yb}^{2+}$. The radically different radiative decay rates of the lowest exciton state and higher excited states enable the generation of a transient photoluminescence enhancement measured via a two-step excitation process. We observe sharp transitions arising from changes of state of localized electrons, broad bands associated with changes of state of delocalized electrons, and broad bands arising from trap liberation.
\end{abstract}

DOI: 10.1103/PhysRevB.84.113110

PACS number(s): 78.47.D-, 71.35.-y, 71.70.Ch, 76.30.Kg

The unique optical properties of rare-earth doped materials are responsible for their crucial role in a wide variety of applications such as fluorescent tubes, white light LEDs, lasers, fiber amplifiers, and medical imaging. ${ }^{1,2}$ The sharp-line optical transitions within the $4 f^{N}(N=0-14)$ ground configurations of rare-earth ions have been extensively studied and may be accurately modeled. ${ }^{1-3}$ Transitions involving excited configurations such as $4 f^{N-1} 5 d$ are crucial to many applications, but information about these states is limited because $4 f^{N} \leftrightarrow$ $4 f^{N-1} 5 d$ spectra consist of broad vibronic bands, yielding much less information than $4 f^{N} \leftrightarrow 4 f^{N}$ spectra. ${ }^{4-8}$ Modern ab initio methods $\mathrm{s}^{9,10}$ are capable of accurately calculating excited-state electronic structure and bond-length variations, giving good agreement with available broad-band $4 f^{N} \leftrightarrow$ $4 f^{N-1} 5 d$ spectra. The $a b$ initio calculations ${ }^{10}$ indicate that transitions between the excited states would give a combination of sharp-line and broad vibronic transitions. We have recently proposed that two-frequency excitation experiments would provide more detailed information about the excited states and better test the calculations. ${ }^{11}$

Excited configurations of rare-earth ions are not restricted to $4 f^{N-1} 5 d$. Configurations involving charge transfer between the rare-earth ion and other ions in the material are also important. Theoretical ${ }^{9}$ and experimental ${ }^{12,13}$ studies suggest that nonradiative relaxation of the $4 f^{N-1} 5 d$ configuration is often mediated by trapped exciton states, where the excited electron is no longer completely localized on the rareearth ion.

In some $\mathrm{Eu}^{2+}$ and $\mathrm{Yb}^{2+}$ materials, emission from excitonic states may be observed. ${ }^{14,15}$ Excitonic emission in $\mathrm{CaF}_{2}$ and $\mathrm{SrF}_{2}$ doped with $\mathrm{Yb}^{2+}$ has been the subject of several studies. ${ }^{14,16-18}$ The ground-state electronic configuration of $\mathrm{CaF}_{2}: \mathrm{Yb}^{2+}$ is $4 f^{14}$. UV excitation can promote one of the $4 f$ electrons to a $5 d$ orbital, giving the excited configuration $4 f^{13} 5 d$. The $5 d$ electron rapidly becomes delocalized over the next-nearest-neighbor $\mathrm{Ca}^{2+}$ ions. The $\mathrm{Yb}^{2+}$ is then effectively ionized to $\mathrm{Yb}^{3+}$, with electronic configuration $4 f^{13}$, i.e., one $4 f$ hole. This trivalent ion attracts the $\mathrm{F}^{-}$nearest neighbors more strongly than a divalent ion, leading to a large contraction of bond length. Emission from the exciton states to the $4 f^{14}$ ground state involves the reverse change in bond length, and therefore a broad, structureless, red-shifted vibrational emission band. ${ }^{14,15}$ Recent $a b$ initio calculations have given valuable insight into the quantum physics of exciton formation. ${ }^{9}$ However, the broad bands provide no detailed information, and experimental information on the energy-level structure of impurity-trapped excitons is largely deduced from indirect measurements such as temperature dependencies, ${ }^{16,17}$ pressure dependencies, ${ }^{12}$ and photoconductivity. ${ }^{18}$

In this Brief Report, we report on an investigation of the internal structure of impurity-trapped excitons using twofrequency measurements of single-crystal $\mathrm{CaF}_{2}$ doped with $\mathrm{Yb}^{2+}$. By applying IR radiation to the crystal after exciting it in the UV, we induce transitions between exciton states. Since some of the exciton-excited states have much higher radiative rates than the lowest exciton state, we can detect the excited state absorption by monitoring photoluminescence enhancement.

$\mathrm{CaF}_{2}: \mathrm{Yb}^{2+}$ crystals were grown using the vertical Bridgmann technique. The UV component of our two-frequency excitation was from a Quantronix TOPAS traveling-wave optical parametric amplifier (OPA) providing 3 ps pulses tunable in the $250-400 \mathrm{~nm}$ region of interest in this work at a repetition rate of $1 \mathrm{kHz}$. Pulsed infrared excitation was achieved using the Dutch free electron laser (FEL) FELIX in Nieuwegein. The IR output of FELIX consists of a 4-6 $\mu$ s macropulse at a repetition rate of $10 \mathrm{~Hz}$, containing micropulses at $25 \mathrm{MHz}$. FELIX is continuously tunable from 3 to $250 \mu \mathrm{m}$. The OPA was synchronized to the FEL, and the electronic timing between the two lasers could be varied. The UV and IR beams were spatially (but not temporally) overlapped on the sample, held at cryogenic temperatures within an Oxford instruments microstat helium flow cryostat. Visible fluorescence was detected using a TRIAX $320 \mathrm{spec}-$ trometer equipped with a $\mathrm{C} 31034$ photomultiplier. Our results for 365-nm-pulsed UV excitation of $\mathrm{CaF}_{2}: \mathrm{Yb}^{2+}$ are consistent 
with previous work, ${ }^{16,17}$ which reported strongly red-shifted fluorescence having a single-exponential decay with a lifetime of $15 \mathrm{~ms}$ at $4.2 \mathrm{~K}$ that reduces at higher temperatures.

In Fig. 1(a), the fluorescence spectra for $\mathrm{CaF}_{2}: \mathrm{Yb}^{2+}$ at $10 \mathrm{~K}$ and $40 \mathrm{~K}$ are shown. At $10 \mathrm{~K}$, the band center and width
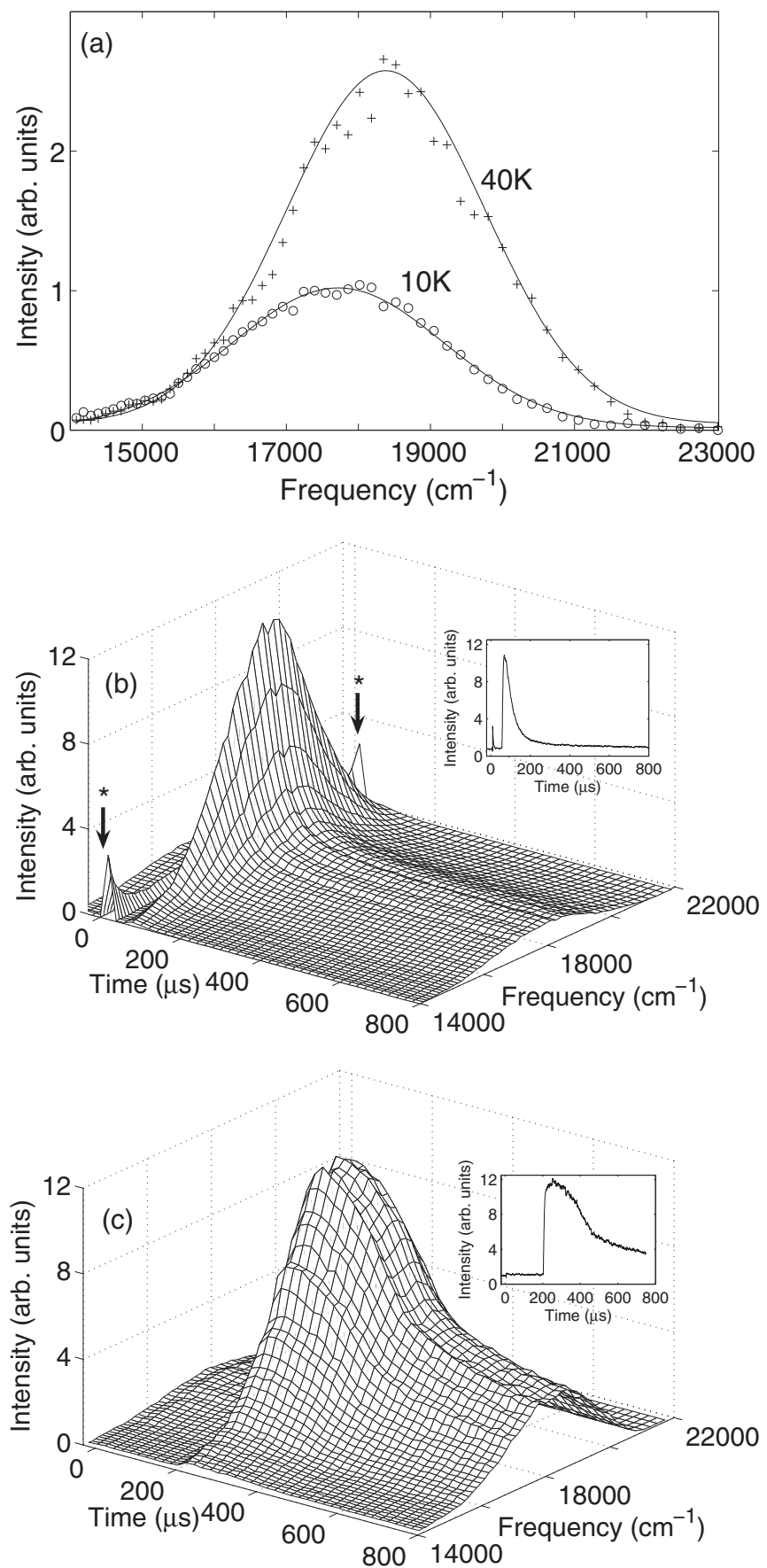

FIG. 1. Fluorescence from $\mathrm{CaF}_{2}: \mathrm{Yb}^{2+}$ for excitation at $365 \mathrm{~nm}$. (a) Spectra at $10 \mathrm{~K}$ and $40 \mathrm{~K} 900 \mu$ s after UV excitation. (b) Time evolution of $10 \mathrm{~K}$ emission spectrum excited with $12.1 \mu \mathrm{m}\left(826 \mathrm{~cm}^{-1}\right)$ IR radiation following the UV pre-pulse. The asterisk $(*)$ shows a combination of first- and second-order UV scatter and emission from minor $\mathrm{Eu}^{2+}$ impurities. (c) Time evolution of $10 \mathrm{~K}$ emission spectrum excited with $16 \mu \mathrm{m}\left(625 \mathrm{~cm}^{-1}\right)$ IR radiation following the UV prepulse. The (b) and (c) insets show the time profile at peak emission wavelength.
(FWHM) are 17720 and $3460 \mathrm{~cm}^{-1}$ respectively. At $40 \mathrm{~K}$, the intensity increases by a factor of 2.5 and the band center and width are 18380 and $3240 \mathrm{~cm}^{-1}$ respectively. Previous analysis of the temperature dependence ${ }^{16}$ suggests that the emission is from two states whose energy separation is about $40 \mathrm{~cm}^{-1}$, with very different radiative lifetimes: $15 \mathrm{~ms}$ for the lower state and $260 \mu$ s for the upper state.

The shift in band center and change in bandwidth indicate that the two excited states have different bond lengths. Emission bandwidths have been previously used to calculate the change in bond length between the lowest exciton state and the ground state as $0.17 \AA,{ }^{16}$ much larger than bondlength changes for transitions between $4 f^{N}$ and $4 f^{N-1} 5 d$ configurations. ${ }^{7,10}$ The calculation is approximate because it uses an effective phonon frequency. A frequency of $325 \mathrm{~cm}^{-1}$ gives a bond-length change of $-0.17 \AA$ for the lowest exciton state relative to the ground state (from our $10 \mathrm{~K}$ data) and $-0.16 \AA$ for the first excited exciton state (from our $40 \mathrm{~K}$ data). The first excited state thus has a longer bond length, closer to the ground state $4 f^{14}$ bond length.

We now present the results of our two-frequency transient measurements at $10 \mathrm{~K}$. Figures 1 (b) and 1(c) show respectively the results of irradiating the system at a wavelength of $12.1 \mu \mathrm{m}$ $\left(826 \mathrm{~cm}^{-1}\right)$ and $16 \mu \mathrm{m}\left(625 \mathrm{~cm}^{-1}\right)$, delayed from the UV excitation by 100-200 $\mu \mathrm{s}$. The application of the IR pulse yields significant enhancement of the emission. Note that the IR pulse on its own cannot induce this optical emission. The enhancement occurs because we now populate excited excitonic states that have significantly higher radiative rates. An enhancement of the total emission at long time scales is also observed. We interpret this as liberation of electrons from traps (discussed below), which is known to occur under intense IR radiation. ${ }^{19}$

For $12.1 \mu \mathrm{m}$ IR excitation, there is a rise time of approximately $6 \mu \mathrm{s}$, and a decay time of $43 \mu \mathrm{s}$. The spectrum at the time corresponding to the maximum emission intensity is similar to the $40 \mathrm{~K}$ spectrum in Fig. 1(a). The enhanced emission is therefore likely to be a result of radiation from the state $40 \mathrm{~cm}^{-1}$ above the lowest exciton state. Further evidence for this conclusion is that at $40 \mathrm{~K}$ the IR excitation gives only a small enhancement and no change to the spectrum. The decay of the transient signal is much faster than the estimated $260 \mu \mathrm{s}$ radiative lifetime, ${ }^{16}$ due to nonradiative processes. ${ }^{17}$

The spectral and temporal behavior for $16 \mu \mathrm{m}$ excitation [Fig. 1(c)] is very different. The time development cannot be fitted by single exponential rise and decay times, but the rise time is approximately $30 \mu \mathrm{s}$ and the decay approximately $160 \mu \mathrm{s}$. At the time corresponding to the maximum emission intensity, we observe a mixture of low-energy and high-energy Gaussians. Several hundred $\mu$ s after the IR excitation pulse, the intensity of the low-energy Gaussian is still significantly higher than before the IR pulse, suggesting that there are significantly more ions radiating than before the IR excitation.

The range of IR energies for which we have observed enhancement of the visible emission is shown in the excitation spectrum of Fig. 2(a), where the photoluminescence transient is time integrated to give the signal. The spectrum consists of a broad band centered at $650 \mathrm{~cm}^{-1}$ and two sharper peaks at $250 \mathrm{~cm}^{-1}$ and $1145 \mathrm{~cm}^{-1}$, with linewidths of 35 and $20 \mathrm{~cm}^{-1}$ respectively. We note that dips in the broad band and the lower 

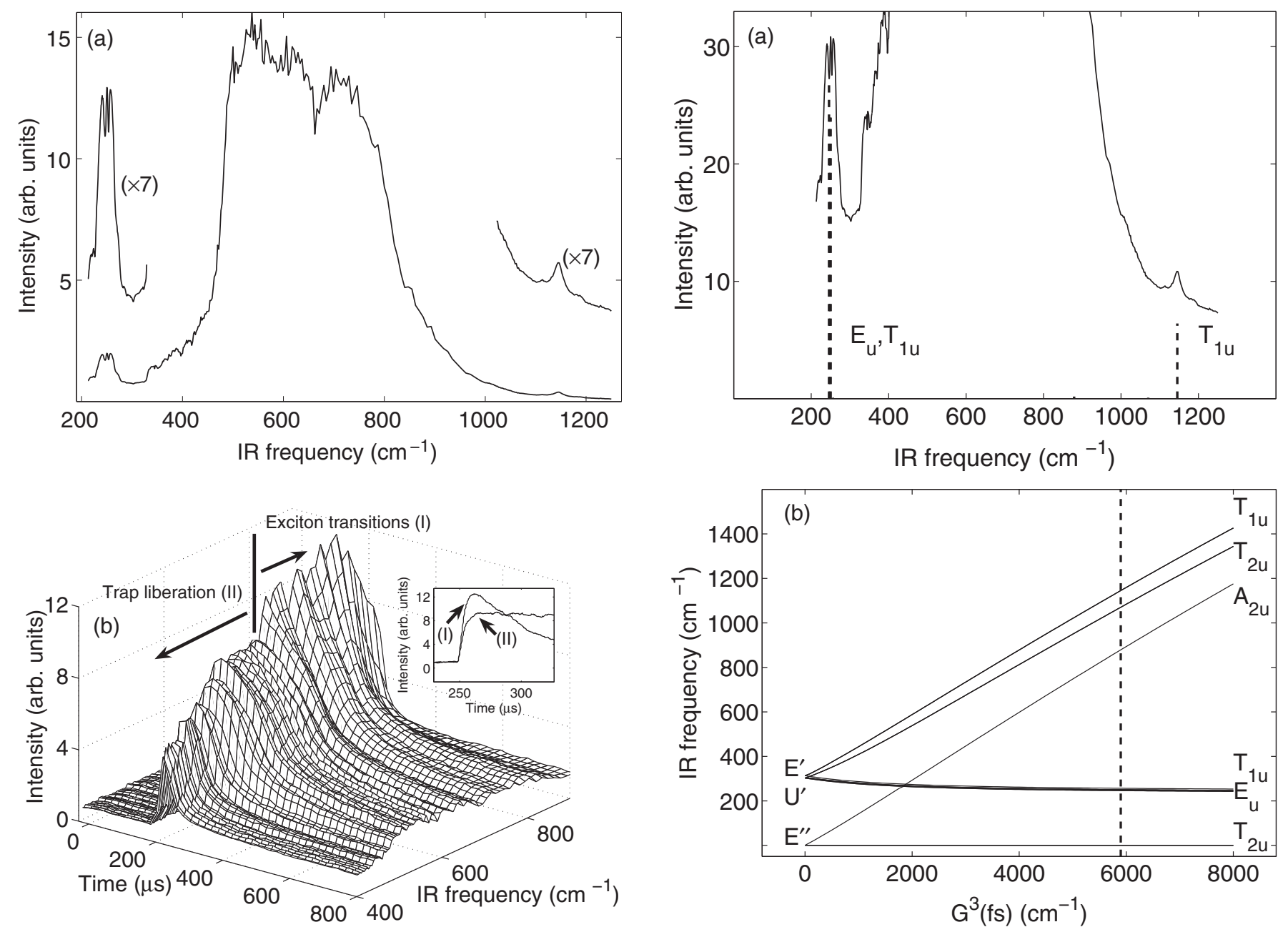

FIG. 2. (a) $10 \mathrm{~K}$ IR excitation spectrum of $\mathrm{CaF}_{2}: \mathrm{Yb}^{2+}$ deduced by integrating emission enhancement over time. The regions where sharp peaks are observed have been magnified and displaced for clarity. (b) Time evolution of the $10 \mathrm{~K} \mathrm{IR}$ excitation spectrum. Inset: rise time with (I) IR excitation at $825 \mathrm{~cm}^{-1}$, (II) IR excitation at $625 \mathrm{~cm}^{-1}$.

sharp peak correlate with atmospheric absorption of the FEL radiation, which can distort the lineshape, despite purging of the IR beam path with dry $\mathrm{N}_{2}$ gas.

The strong dependence of the temporal dynamics on the IR excitation wavelength is further illustrated in Fig. 2(b). The broad peak is actually two distinct regions, a higher-energy region with fast rise and decay times, and a lower-energy region dominated by slow rise and decay times. We attribute the long-lived signal in this region to trap liberation processes. Whereas transitions within the exciton transfer population from the lowest exciton state to a state with a much higher radiative rate, trap liberation processes increase the total number of excitons radiating. A phenomenological model of a Coulomb trap having a threshold of $380 \mathrm{~cm}^{-1}$ gives an asymmetric spectrum with a width of approximately $400 \mathrm{~cm}^{-1}$ [Ref. 19, Eq. (3)], which is consistent with the width of the low-energy band.

The high-energy part of the band, from 650 to $950 \mathrm{~cm}^{-1}$, and the sharp lines, are assigned to transitions within the exciton. Recall that our exciton model is a $\mathrm{Yb}^{3+}$ ion with one $4 f$ hole $\left(4 f^{13}\right)$ and a delocalized electron. It is the

FIG. 3. (a) Calculated oscillator strengths (vertical lines) and experimental IR excitation spectrum. (b) Dependence of the exciton energy levels on the exchange parameter $G^{3}(f s)$. The vertical dotted line is at the value of $G^{3}(f s)$ used in the calculation of Fig. 3(a).

delocalized electron that can affect bonding, so the vibronic broadening of the $650-950 \mathrm{~cm}^{-1}$ band leads us to attribute this feature to transitions that change the orbital of the delocalized electron and therefore the bond length. The width of this band is similar to the width of the phonon spectrum in $\mathrm{CaF}_{2},{ }^{20}$ which implies that the bond-length change for the transition is small, similar to the difference in bond length between the lowest two exciton states calculated above $(0.01 \AA)$. An accurate calculation of the position and width of this band should be possible with a detailed $a b$ initio approach, as in Ref. 9.

The sharp excitation features observed at 250 and $1145 \mathrm{~cm}^{-1}$ cannot involve a change in bonding. We therefore assign them to changes in the wave function of the localized $4 f$ hole or the relative spin of the $4 f$ hole and delocalized electron. The latter is associated with an exchange Coulomb interaction. The calculations of Ref. 9 suggest that the excitons involve a linear combination of $5 d$ and $6 s$ orbitals with totally symmetric $s$ character. Unlike the $\mathrm{SrCl}_{2}: \mathrm{Yb}^{2+}$ system, ${ }^{10}$ detailed calculations for $\mathrm{CaF}_{2}: \mathrm{Yb}^{2+}$ are not available. However, we may model the sharp lines with a simple semiempirical 
model by constructing a "crystal field" Hamiltonian for an $s$ electron and a $4 f$ hole in a cubic crystal field,

$$
\begin{aligned}
H_{\mathrm{cf}}= & \zeta A_{\mathrm{so}}+B^{4}\left(C_{0}^{4}+\sqrt{\frac{5}{14}}\left[C_{4}^{4}+C_{-4}^{4}\right]\right) \\
& +B^{6}\left(C_{0}^{6}-\sqrt{\frac{7}{2}}\left[C_{4}^{6}+C_{-4}^{6}\right]\right)+G^{3}(f s) g_{3}(f s),
\end{aligned}
$$

where $\zeta$ is the spin-orbit interaction for the $4 f$ electrons, $B^{4}$ and $B^{6}$ are crystal-field parameters for the $4 f$ electrons, and $G^{3}(f s)$ is the exchange interaction between the $4 f$ and the delocalized electron. Details of the Hamiltonian operators may be found in Refs. 3,4,6,8,21.

Since we only observe two sharp lines, it is not possible to fit all of the parameters in Eq. (1). The spin-orbit parameter $\zeta$ was fixed at $2928 \mathrm{~cm}^{-1}$, the value determined for $\mathrm{Yb}^{3+}$ in $\mathrm{LaF}_{3} .^{3}$ The ratio $B^{6} / B^{4}$ was fixed at -0.35 , the value obtained by Leśniak ${ }^{22}$ for $\mathrm{Er}^{3+}$ in $\mathrm{CaF}_{2}$. With these assumptions, the parameter values $B^{4}=-800 \mathrm{~cm}^{-1}$ and $G^{3}(f s)=5900 \mathrm{~cm}^{-1}$ give energy levels that match the observed sharp lines.

Figure 3 illustrates our calculation. When the $G^{3}(f s)$ parameter is set to zero, the ${ }^{2} F_{7 / 2}$ multiplet of $4 f^{13}$ splits into $E^{\prime \prime}, U^{\prime}$, and $E^{\prime}$ irreducible representations of the octahedral group, ${ }^{23}$ as illustrated on the left side of Fig. 3(b). As $G^{3}(f s)$ is increased, these states couple with ${ }^{2} S$ (from the $s$ electron). In cubic symmetry, only magnetic-dipole transitions are allowed. Calculated absorption oscillator strengths are compared with experiment in Fig. 3(a). Note that several transitions are forbidden or calculated to be very small.
The $B^{4}$ parameter is similar to the value of $-725 \mathrm{~cm}^{-1}$ for the $4 f^{13} 5 d$ configuration of $\mathrm{Yb}^{2+}$ in $\mathrm{SrCl}_{2} \cdot{ }^{8}$ A Hartree-Fock calculation using the atomic code of Cowan $^{21}$ gives $G^{3}(f s)=$ $3167 \mathrm{~cm}^{-1}$ for the $4 f^{13} 6 s$ configuration of $\mathrm{Yb}^{2+}$. This is smaller than our experimental value. However, the calculations of Ref. 9 suggest that the exciton will contain mixtures of $5 d$ and $6 s$ orbitals. The exchange splitting for $4 f^{13} 5 d$ is over $2000 \mathrm{~cm}^{-1}, 8$ so our observed splitting of about $1145 \mathrm{~cm}^{-1}$ for the exciton, where the electron is in a more delocalized orbital than $5 d$, is reasonable.

In conclusion, we have demonstrated that it is possible to probe the internal structure and dynamics of exciton states, and trap-liberation processes, in rare-earth materials with a two-frequency selective fluorescence enhancement technique. We observe sharp-line transitions within the exciton spectrum. A quantitative account of these sharp line features has been obtained using a parametrized crystal-field model. Our observations provide a basis and motivation for detailed $a b$ initio calculations as in Ref. 9 and for investigation of other rare-earth excitonic systems using the same experimental approach.

This work was supported by the Marsden fund of the Royal Society of New Zealand, Grant No. 09-UOC-080. We thank the Dutch FOM organization for providing FELIX beamtime and the FELIX staff for their assistance. P.S.S acknowledges support from the University of Canterbury. C.-K.D. acknowledges support from the Natural Science Foundation of China, Grant No. 11074315.
*Corresponding author: jon-paul.wells@ canterbury.ac.nz

${ }^{1}$ Properties of Rare Earths in Optical Materials, edited by G. K. Liu and B. Jacquier (Springer, Berlin, 2005).

${ }^{2}$ Luminescence: From Theory to Applications, edited by C. Ronda (Wiley-VCH, Weinheim, 2007).

${ }^{3}$ W. T. Carnall, G. L. Goodman, K. Rajnak, and R. S. Rana, J. Chem. Phys. 90, 3443 (1989).

${ }^{4}$ L. van Pieterson, M. F. Reid, R. T. Wegh, S. Soverna, and A. Meijerink, Phys. Rev. B 65, 045113 (2002).

${ }^{5}$ L. van Pieterson, M. F. Reid, G. W. Burdick, and A. Meijerink, Phys. Rev. B 65, 045114 (2002).

${ }^{6} \mathrm{G}$. W. Burdick and M. F. Reid, in Handbook on the Physics and Chemistry of the Rare Earths, Vol. 37, edited by K. A. Gschneidner Jr., J. C. Bunzli, and V. K. Percharsky (Elesevier, North Holland, 2007), Chap. 232, pp. 61-91.

${ }^{7}$ M. Karbowiak, A. Urbanowicz, and M. F. Reid, Phys. Rev. B 76, 115125 (2007).

${ }^{8}$ Z. Pan, C. K. Duan, and P. A. Tanner, Phys. Rev. B 77, 085114 (2008).

${ }^{9}$ G. Sánchez-Sanz, L. Seijo, and Z. Barandiarán, J. Chem. Phys. 133, 114509 (2010).

${ }^{10}$ G. Sánchez-Sanz, L. Seijo, and Z. Barandiarán, J. Chem. Phys. 133, 114506 (2010).
${ }^{11}$ M. F. Reid, L. Hu, S. Frank, C. K. Duan, S. Xia, and M. Yin, Eur. J. Inorg. Chem. 2010, 2649 (2010).

${ }^{12}$ M. Grinberg and S. Mahlik, J. Non-Cryst. Solids 354, 4163 (2008).

${ }^{13}$ S. Mahlik, M. Grinberg, E. Cavalli, M. Bettinelli, and P. Boutinaud, J. Phys.: Condens. Matter 21, 105401 (2009).

${ }^{14}$ D. S. McClure and C. Pedrini, Phys. Rev. B 32, 8465 (1985).

${ }^{15}$ P. Dorenbos, J. Phys. Condens. Matter 15, 2645 (2003).

${ }^{16}$ B. Moine, B. Courtois, and C. Pedrini, J. Phys. 50, 2105 (1989).

${ }^{17}$ B. Moine, B. Courtois, and C. Pedrini, J. Lumin. 48-49, 501 (1991).

${ }^{18}$ C. Pedrini, M. F. Joubert, and D. S. McClure, J. Lumin. 125, 230 (2007).

${ }^{19}$ I. Izeddin, M. A. J. Klik, N. Q. Vinh, M. S. Bresler, and T. Gregorkiewicz, Phys. Rev. Lett. 99, 077401 (2007).

${ }^{20}$ W. Hayes, M. C. K. Wiltshire, W. J. Manthey, and D. S. McClure, J. Phys. C 6, L273 (1973).

${ }^{21}$ R. D. Cowan, The Theory of Atomic Structure and Spectra (U. California, Berkeley, 1981).

${ }^{22}$ K. Leśniak, J. Phys. Condens. Matter 2, 5563 (1990).

${ }^{23}$ B. Henderson and G. F. Imbusch, Optical spectroscopy of inorganic solids (Clarendon Press, Oxford, 1989). 